\title{
Organelle_PBA, a pipeline for assembling chloroplast and mitochondrial genomes from PacBio DNA sequencing data
}

\author{
Aboozar Soorni ${ }^{1,2}$, David Haak ${ }^{3}$, David Zaitlin ${ }^{4}$ and Aureliano Bombarely ${ }^{1 *}$ (D)
}

\begin{abstract}
Background: The development of long-read sequencing technologies, such as single-molecule real-time (SMRT) sequencing by PacBio, has produced a revolution in the sequencing of small genomes. Sequencing organelle genomes using PacBio long-read data is a cost effective, straightforward approach. Nevertheless, the availability of simple-to-use software to perform the assembly from raw reads is limited at present.

Results: We present Organelle-PBA, a Perl program designed specifically for the assembly of chloroplast and mitochondrial genomes. For chloroplast genomes, the program selects the chloroplast reads from a whole genome sequencing pool, maps the reads to a reference sequence from a closely related species, and then performs read correction and de novo assembly using Sprai. Organelle-PBA completes the assembly process with the additional step of scaffolding by SSPACE-LongRead. The program then detects the chloroplast inverted repeats and reassembles and re-orients the assembly based on the organelle origin of the reference. We have evaluated the performance of the software using PacBio reads from different species, read coverage, and reference genomes. Finally, we present the assembly of two novel chloroplast genomes from the species Picea glauca (Pinaceae) and Sinningia speciosa (Gesneriaceae).
\end{abstract}

Conclusion: Organelle-PBA is an easy-to-use Perl-based software pipeline that was written specifically to assemble mitochondrial and chloroplast genomes from whole genome PacBio reads. The program is available at https://github.com/aubombarely/Organelle_PBA.

Keywords: Chloroplast, Mitochondria, PacBio, Organelle Genome Assembly

\section{Background}

Single Molecule Real Time (SMRT) sequencing technology developed by Pacific Biosciences (PacBio), can produce millions of long reads $(1 \mathrm{~Kb}$ or longer, with a current average of $12 \mathrm{~Kb}$ ) per run. SMRT sequencing is based on single molecule real-time imaging of the incorporation of fluorescently tagged nucleotides to a DNA template molecule [1]. This technology has been successfully applied to a wide range of experiments and species such as the sequencing of DNA amplicons [2] and transcriptomes [3]. Nevertheless the most popular application is whole genome sequencing. It has been used for the sequencing of bacterial genomes such as the plant

\footnotetext{
*Correspondence: aurebg@vt.edu

'Department of Horticulture, Virginia Polytechnic Institute and State

University, Blacksburg, VA 24061, USA

Full list of author information is available at the end of the article
}

pathogen Xanthomonas oryzae [4]. PacBio reads have also been used for the sequencing of complex plant nuclear genomes, such as that of the Adzuki bean, Vigna angularis [5], demonstrating the advantage of this technology for resolving repetitive regions during sequence assembly.

Sequence assembly is the process whereby one or more consensus sequences are reconstructed from hundreds to billions of individual DNA sequence reads. Although there are dozens of programs to produce consensus sequences, they can be classified into two groups based on the algorithm they use: Overlap-Layout-Consensus (OLC) and De Bruijn Graph (DBG). OLC algorithms are best suited for low coverage long read approaches. The most popular PacBio assemblers such as HGAP [6] and Falcon (https:// github.com/PacificBiosciences/falcon) utilize OLC algorithms. A popular OLC-based program, the Celera Assembler (CA; [7]), has been updated to assemble PacBio reads 
[8] (this program, called PBcR, is being replaced by Canu [http://canu.readthedocs.org/] and is no longer maintained). Another option is the use of Sprai [9], a pipeline that employs the CA assembly algorithm. This pipeline pre-selects the best $20 \mathrm{X}$ coverage reads using BLAST searches [10], then corrects and assembles them using CA. PacBio sequencing and OLC assemblers have been successfully applied to the sequencing of yeast mitochondrial genomes [11] and chloroplast genomes such as those of Potentilla micrantha [12], Nelumbo nucifera [13], and sugar beet (Beta vulgaris) [14].

Mitochondrial and chloroplast DNA markers are the bridge between population genetics and systematics, primarily because they are maternally inherited and do not recombine; thus they can facilitate the reconstruction of maternal lineages [15]. Mitochondrial genomes vary in size depending on the eukaryotic lineage. For animals, the lengths range from 28,757 bp (Breviceps adspersus) [16] to 13,424 bp (Didemnum vexillum) [17] with the average size being $16,800 \mathrm{bp}$. Conversely, mitochondrial genomes of plants and fungi can vary by almost three orders of magnitude, ranging from 15,758 bp (Chlamydomonas reinhardtii) [18] to $11.3 \mathrm{Mb}$ (Silene conica) [19]. Chloroplast genomes, on the other hand, are typically much more conserved in their size and structure, ranging from 11,348 bp (Pilostyles aethopica) [20] to 521,168 bp (Floydiella terrestris) [21], with an average size of ca. 148,000 bp. At present, $>10,000$ mitochondrial genomes have been sequenced, while comparatively fewer $(\sim 990)$ chloroplast genomes have been sequenced (http://www.ncbi.nlm.nih.gov/genome).

The application of PacBio long-read DNA sequencing technology to organelle genome sequencing will duplicate the numbers given above in the next 2 years. As previously described, there are several tools designed to assemble PacBio reads (e.g. HGAP, Falcon, Canu, and Sprai); however, no single tool is available to assemble organelle genomes using total DNA sequencing reads derived from the PacBio platform. We have developed a new Perl-based tool, Organelle_PBA, designed expressly to reconstruct whole organelle genomes from PacBio data. First, the program selects the specific organelle reads by mapping raw reads to a reference organelle genome. Then, it produces a de-novo assembly using Sprai, a new re-scaffolding program, and removes the redundancy produced by the circular organization of these genomes. Organelle_PBA also resolves the inverted repeats found in chloroplast genomes. The tool is available at https://github.com/aubombarely/Organelle_PBA.

\section{Material and methods}

PacBio reads from Arabidopsis thaliana (SRR1284093, SRR1284094, SRR1284095, SRR1284703, SRR1284704), Mus musculus (ERR731675) and Picea glauca (SRR2148116) were downloaded from the SRA repository using the Prefech program from the SRA Toolkit. The SRA file format was then converted to Fastq format using the Fastq-dump program in the SRA Toolkit.

Sinningia speciosa PacBio reads were obtained by de novo PacBio DNA sequencing. Briefly, the S. speciosa variety 'Avenida Niemeyer' [22] was grown under fluorescent lighting at room temperature $\left(\sim 23{ }^{\circ} \mathrm{C}\right)$. DNA was extracted from young flower buds using the Qiagen DNEasy $^{\circ}$ extraction kit. DNA was quantified using a Nanodrop ${ }^{\oplus}$ ND-1000 spectrophotometer, and its integrity was evaluated by agarose gel electrophoresis. DNA was sent to the Duke Center for Genomics and Computational Biology facility, where a SMRTBell ${ }^{\mathrm{TM}}$ long insert PacBio library $(15-20 \mathrm{~Kb}$ fragments) was prepared and then sequenced using a PacBio RSII system (P6-C4 Chemistry). PacBio reads were used directly in Organelle_PBA without any extra processing.

Organelle reference genomes were downloaded from the NCBI nucleotide database. The downloaded references were M. musculus, NC_005089.1; Mus carolis, NC_ 025268.; Rattus norvegicus, NC_001665.2; Marmota himalayana, NC_018367.1; A. thaliana, NC_000932.1 and NC_001284.2; Brassica napus, NC_016734.1; Vitis vinifera, NC_007957.1; P. abies NC_021456.1 and Boea hygrometrica NC_016468.1.

For organelle genome coverage evaluation, the PacBio reads were mapped using BlasR [23] with the sam output format parameter. The result was piped into SAMtools for filtering of the unmapped reads [24]. Coverage was calculated using BEDtools [25], and variants, SNPs, and InDels were called using FreeBayes [26].

\section{Implementation}

Organelle_PBA is a program written in Perl constructed as a single file script. It uses the following Perl 5.18 modules: Getopts::Std (core), File::Spec (core), File::Basename (core), File::Copy (core), File::Path (core), IPC::Cmd (core), Math::BigFloat (core), Bio::SeqIO (bioperl) and Bio::Tools::Run::StandAloneBlastPlus (bioperl). Additionally, it uses the following programs: BlasR [23], SAMtools [24], Blast [10], SSPACE-LongRead [27], Sprai [9] and BEDtools [25]. The executable files are passed to the script as environmental variables if they are not accessible from the PATH. Upon initial run, the script checks that all of the executable files are available prior to initializing any processes. The subprograms are run using the IPC::Cmd Perl module.

The program is divided into the following steps (Additional file 1: Figure S1):

0. Argument check and analysis of the input stats such as organelle reference size and number of reads. 
1. Mapping of the PacBio reads to the organelle reference genome using BlasR.

2. Parsing of the BlasR results and selection of the PacBio reads that map to the organelle reference. The percentage of the read length aligned to the reference can be used to filter these hits.

3. Read correction and assembly using Sprai using the reads selected in the previous step. Reads can be filtered by length before the assembly using the Sprai arguments.

4. Assembly evaluation comparing the total assembly size and the longest contig size with the reference sequence size. If the longest contig is longer than the reference genome, the script moves to step 6 (circular assembly check), otherwise it continues to step 5 .

5. If the longest contig size is smaller than the reference, Organelle_PBA interprets the assembly as being fragmented. It then runs BlasR and SSPACE-LongRead with the entire read dataset to find any reads that it could not select during the BlasR mapping (Step 1). After this, it evaluates the assembly again reporting the new sizes and then moving to step 6 .

6. Circular assembly check by homology search (Blast) of the assembled sequence with itself. The program also checks for a possible origin based on the reference through a homology search (Blast). If it finds any of these, it will break the contig/scaffold, reorganizing the pieces to remove the redundancy from a circular assembly and orient the assembly based on the reference genome sequence.

7. Check the completeness of the assembly. Chloroplast genomes are composed of four parts:
Long Single Copy (LSC) section, Short Single Copy (SSC), and two Inverted Repeats: IRa and IRb. The inverted repeats, IRa and IRb, are identical and sometimes are only partially assembled, so the assembly could appear to be complete with a size smaller than the reference. If this is the case, the program will move to step 8 , if it is not, it will move to step 9.

8. Inverted repeat evaluation and resolution. Organelle_PBA will map all the reads back to the assembly using BlasR to calculate the coverage for each part of the assembly. Inverted repeats appear with twice the coverage of the non-repeated region as a result of the multiple mapping sites reported by BlasR (see Results). Additionally, the program will break these regions looking for sequence homology using Blast to analyze if they present a certain level of homology reported by BlastN. If the script finds it, it will remove the redundancy and rebuild the assembly using SSPACE-LongRead.

9. Final assembly analysis and assembly statistics report printing.

\section{Results \\ Mus musculus mitochondrial genome assembly}

Sets of 50,000, 100,000, and 163,477 randomly selected PacBio reads from the house mouse, Mus musculus (SRA datatset: ERR731675), were used to test the mitochondrial genome assembly using different mitochondrial reference genomes; M. musculus, NC_005089.1 (same species); Mus carolis, NC_025268.1 (same genus); Rattus norvegicus, NC_001665.2 (same family - Muridae) and Marmota himalayana, NC_018367.1 (different family - Sciuridae). Results are summarized in the Table 1.

Table 1 Summary of the M. musculus mitochondrial genome assembly

\begin{tabular}{|c|c|c|c|c|c|c|}
\hline Input reads & Reference & Mapped reads & \% Bases mapped & Estimated depth $(x)$ & Assembly size (bp) & Organelle completed $^{a}$ \\
\hline 50,000 & Mus musculus & 39 & 0.22 & 26 & 2678 & $\mathrm{NO}$ \\
\hline 100,000 & & 83 & 0.18 & 42 & 12,377 & NO \\
\hline 163,477 & & 138 & 0.22 & 69 & 16,294 & YES \\
\hline 50,000 & Mus caroli & 35 & 0.20 & 24 & NA & NO \\
\hline 100,000 & & 69 & 0.16 & 36 & 12,332 & NO \\
\hline 163,477 & & 110 & 0.15 & 56 & 16,299 & YES \\
\hline 50,000 & Rattus norvegicus & 26 & 0.16 & 19 & NA & NO \\
\hline 100,000 & & 53 & 0.13 & 30 & 10,247 & NO \\
\hline 163,477 & & 86 & 0.12 & 44 & 16,292 & YES \\
\hline 50,000 & Marmota himalayana & 10 & 0.07 & 8 & NA & NO \\
\hline 100,000 & & 17 & 0.05 & 11 & 6580 & NO \\
\hline 163,477 & & 31 & 0.04 & 17 & 7193 & NO \\
\hline
\end{tabular}

${ }^{a}$ The mitochondria genome assembly was considered complete when the difference in size compared to the reference genome was $<10$ nucleotides 
The different read sets represented 26X, 42X, and 69X sequencing depth, respectively, for the $M$. musculus mitochondrial genome $(\sim 0.2 \%$ of the downloaded PacBio dataset). Organelle_PBA produced a complete M. musculus mitochondrial genome assembly for the 163,477 reads set (69X) with all of the reference sequences except for the $M$. himalayana mitochondrial genome. The average running time for this process was $117 \mathrm{~s}$. The PacBio read remapping showed that the assembly was fully covered (from $\sim 5 \mathrm{X}$ to $\sim 25 \mathrm{X}$ ) with no gaps (Fig. 1a and b). Additionally, a comparison of the assembled mitochondrial genome with the $M$. musculus mitochondrial reference (NC_005089.1) showed a perfect alignment with 395 SNPs distributed across the entire assembly (Fig. 1c).
To compare the performance of this approach compared with a whole dataset assembly and posterior identification of the organelle genome, we performed a whole dataset assembly using Canu and Sprai using similar configuration parameters. Canu assembled the 163,477 reads in $3970 \mathrm{~s}$ producing 7 contigs with a $\mathrm{L} 50=17,784 \mathrm{bp}$. We identified a 15,206 bp mitochondrial genome contig by BlastN homology search. Sprai assembled the same dataset in $3660 \mathrm{~s}$ producing 5 contigs with a $\mathrm{L} 50=5858 \mathrm{bp}$. None of the Sprai contigs were identified as the mitochrondrial genome.

Based on these results, we can conclude that $>50 \mathrm{X}$ sequencing depth and a reference genome sequence from the same taxonomic family is recommended for the assembly of a full mammalian mitochondrial genome.

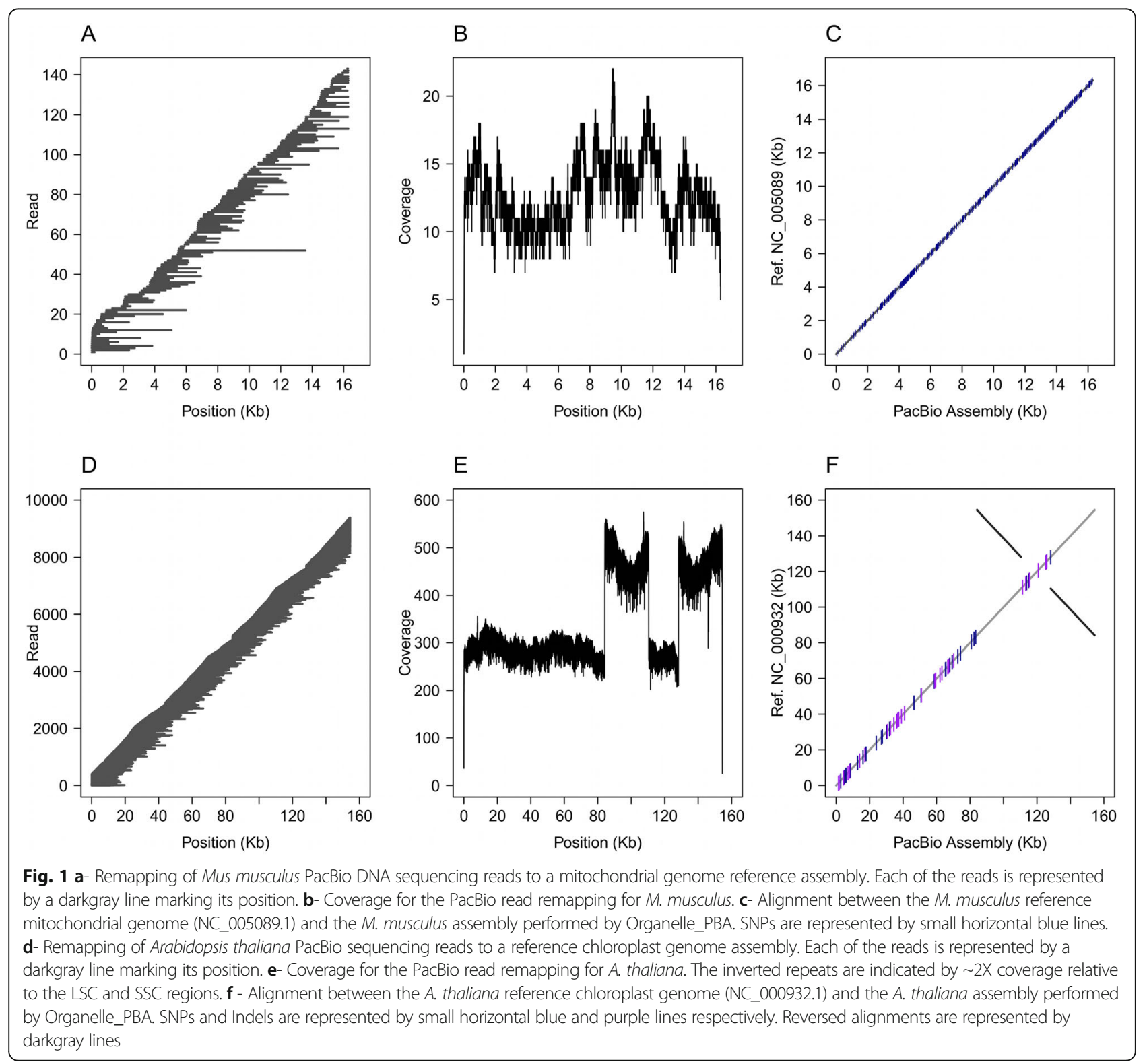




\section{Arabidopsis thaliana chloroplast genome assembly}

Sets of 5000, 10,000, 50,000, 100,000, and 163,448 randomly selected PacBio reads from Arabidopsis thaliana (SRA dataset: SRR1284093) were used to test the chloroplast genome assembly using different chloroplast references; A. thaliana, NC_000932.1 (same species); Brassica napus, NC_016734.1 (same family); and Vitis vinifera, NC_007957.1 (family Vitaceae). Results are summarized in Table 2. The different read sets represented 23X, 50X, 244X, 470X, and 771X sequencing depth for the $A$. thaliana chloroplast genome, respectively ( $23 \%$ of the downloaded PacBio dataset). The running time for the whole set $(163,448$ reads) was 5234 s. The assembly was fully covered with no gaps (Fig. 1d), and showed coverage ranging from $\sim 50 \mathrm{X}$ to $\sim 600 \mathrm{X}$. The inverted repeats showed twice the average coverage of the LSC and SSC regions (Fig. 1e). The comparison with the $A$. thaliana reference (NC_000932.1) showed 37 SNPs, 53 insertions, and 73 deletions in the LSC and SSC regions (Fig. 1f).

Additionally we performed the whole dataset assembly and posterior chloroplast identification by BlastN sequence homology to compare with our approach where the read identification is performed before the assembly. Canu produced 31 contigs in $6040 \mathrm{~s}$ with a L50 of $32,701 \mathrm{bp}$. We identified three chloroplastic contigs with lengths of $117,666,53,847,30,575$ bp respectively. Sprai produced 2 contigs in 8591 s. The longest sequence represented the complete chloroplast genome with a length of 163,611 bp including a redundant region of 9133 bp caused by the circularity of the chloroplast genome.
Based on these results, we conclude that it is necessary to have at least 200X sequencing depth to obtain a fully assembled chloroplast genome. Considering the taxonomic distance of the reference sequence, we can suggest that almost any chloroplast genome from a species in the same subclass should be usable in selecting the chloroplast reads. We did not test the use of chloroplast reference genomes from other subclasses (e.g. an asterid reference to assemble the rosid $A$. thaliana chloroplast genome) because there are enough reference genomes from the same subclass publically available. The use of a whole dataset assembly with Sprai and posterior identification delivered the complete chloroplast genome sequence, although this approach used $64 \%$ more time without counting a final result curation to remove the redundant region.

\section{Arabidopsis thaliana mitochondrial genome assembly}

Sets of 5000, 10,000, 50,000, 100,000, 163,448, 490,143, and 817,099 randomly selected PacBio reads from Arabidopsis thaliana (SRA datasets: SRR1284093, SRR1284094, SRR1284095, SRR1284703 and SRR1284704) were used to test the mitochondrial genome assembly using the $A$. thaliana mitochondrial reference sequence (NC_001284.2). The results of the assemblies are summarized in Table 3. No complete mitochondrial genome assembly was obtained using this methodology. The mapping of the full $A$. thaliana dataset SRR1284093 (163,448 reads) to the $A$. thaliana mitochondrial genome NC_001284.2 selected 3046 reads (equivalent to $111 \mathrm{X}$ ) but a close inspection showed an average coverage of $9 \mathrm{X}$ with 14,533 non-covered positions;

Table 2 Summary of the A. thaliana chloroplast genome assembly

\begin{tabular}{|c|c|c|c|c|c|c|}
\hline Input reads & Reference & Mapped reads & \% Bases mapped & Estimated depth $(x)$ & Assembly size (bp) & Organelle completed $^{a}$ \\
\hline 5000 & Arabidopsis thaliana & 287 & 23.06 & 23 & 42,978 & $\mathrm{NO}$ \\
\hline 10,000 & & 611 & 24.40 & 50 & 150,039 & $\mathrm{NO}$ \\
\hline 50,000 & & 3,013 & 23.79 & 244 & 154,472 & YES \\
\hline 100,000 & & 5,777 & 23.01 & 470 & 154,471 & YES \\
\hline 163,448 & & 9,409 & 23.15 & 771 & 154,474 & YES \\
\hline 5000 & Brassica napus & 277 & 21.82 & 22 & 59,513 & $\mathrm{NO}$ \\
\hline 10,000 & & 591 & 23.08 & 48 & 153,132 & $\mathrm{NO}$ \\
\hline 50,000 & & 2923 & 23.08 & 239 & 154,474 & YES \\
\hline 100,000 & & 5565 & 22.13 & 457 & 154,481 & YES \\
\hline 163,448 & & 9102 & 22.43 & 755 & 154,473 & YES \\
\hline 5000 & Vitis vinifera & 233 & 18.88 & 18 & 73,382 & $\mathrm{NO}$ \\
\hline 10,000 & & 507 & 20.62 & 41 & 151,984 & $\mathrm{NO}$ \\
\hline 50,000 & & 2516 & 20.67 & 204 & 154,469 & YES \\
\hline 100,000 & & 4807 & 20.04 & 393 & 154,477 & YES \\
\hline 163,448 & & 7855 & 20.28 & 649 & 154,472 & YES \\
\hline
\end{tabular}

${ }^{\mathrm{a}}$ The chloroplast genome assembly was considered complete when the difference in size compared to the reference genome was $<10$ nucleotides 
Table 3 Summary of the A. thaliana mitochondrial genome assembly

\begin{tabular}{|c|c|c|c|c|c|c|}
\hline Input reads & Reference & Mapped reads & $\%$ Bases mapped & Estimated depth $(\mathrm{x})$ & Assembly size (bp) & Organelle completed ${ }^{a}$ \\
\hline 5000 & Arabidopsis thaliana & 101 & 8.88 & 4 & 27,294 & NO \\
\hline 10,000 & & 215 & 8.80 & 8 & 57,303 & NO \\
\hline 50,000 & & 1,006 & 8.63 & 37 & 156,177 & NO \\
\hline 100,000 & & 1,861 & 7.92 & 68 & 152,405 & NO \\
\hline 163,448 & & 3,046 & 7.92 & 111 & 177,810 & NO \\
\hline 490,143 & & 11,080 & 8.31 & 434 & 136,334 & NO \\
\hline 817,099 & & 21,099 & 8.72 & 829 & 150,873 & NO \\
\hline
\end{tabular}

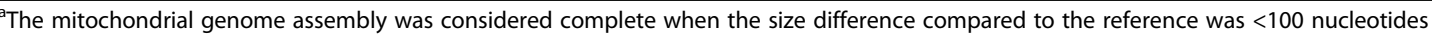

thus, the selection of mapped reads for this dataset does not represent the full mitochondrial genome. Increasing the number of reads to 490,143 and 817,099 also increased the effective coverage to $33 \mathrm{X}$ and $64 \mathrm{X}$, but there were still 8045 and 6305 non-covered positions, respectively, that produced incomplete assemblies.

\section{Novel organelle genome assemblies}

To test the ability of Organelle_PBA to assemble new plant organelle genomes, we performed chloroplast genome assemblies for two species Picea glauca (white spruce) and Sinningia speciosa (a wild form of the cultivated florist's gloxinia). For P. glauca, a read dataset with 563,675 reads was downloaded from the SRA repository (SRR2148116). For Sinninigia speciosa, a set of 923,290 reads was selected from the $S$. speciosa whole genome sequencing project (manuscript in preparation). There is no chloroplast genome assembly available for P. glauca, but there is a chloroplast genome reference from a species in the same genus, $P$. abies (NC_021456.1). The $P$. glauca chloroplast assembly produced a $123,423 \mathrm{bp}$ contig with 237 nucleotide insertions, 302 nucleotide deletions, and 416 SNPs compared with the P. abies chloroplast genome. For the $S$. speciosa chloroplast genome assembly we used the Boea hygrometrica chloroplast genome (same family as S. speciosa, but different subfamily). The $S$. speciosa assembly produced a 153,428 bp contig with 2212 nucleotide insertions, 1766 nucleotide deletions, and 6783 SNPs compared with the $B$. hygrometrica chloroplast genome sequence.

\section{Discussion}

Organelle_PBA is a script designed to assemble organelle genomes from long-read whole genome sequencing data by selecting the organelle reads and then mapping them to a closely related reference sequence. Animal mitochondria and plant chloroplast genomes are generally highly conserved across different lineages, so the use of a reference sequence from the same family is usually enough to select reads and then perform a focused assembly, thereby reducing the use of computational resources. Our results indicate that coverage between 50 and
200X is usually enough to obtain a full organelle genome assembly. Our analysis shows that an average whole genome sequencing project contains $\sim 0.2 \%$ of animal mitochondrial DNA and $\sim 20 \%$ of plant chloroplast DNA, which, in most cases, is enough to reach $>50 \mathrm{X}$ organelle genome coverage. Mapping of these reads shows that they are equally distributed across the organelle genome such that the mapping strategy employed effectively captures the full representation of the organelle genome. Nevertheless, the assembly of plant mitochondrial genomes can be difficult because they are more variable in size and genomic composition, and they are usually poorly represented in whole genome sequencing datasets. The assembly of the A. thaliana mitochondrial genome was incomplete, likely due to incomplete mapping to the reference genome. Additionally, Sprai selects only the best 20X coverage of the PacBio reads to perform the assembly, so unequal mapping could introduce bias into the Sprai read selection. We also found, through coverage analysis, two peaks of high coverage $(>1000 \mathrm{X})$ that probably represent highly repetitive regions, although a more detailed analysis needs to be performed to verify this hypothesis.

Finally, even if assembly of an organelle genome is not the final goal of a whole genome sequencing project, there are some advantages to assembling the organelle genome prior to launching the nuclear genome assembly: 1) It can facilitate assembly of the nuclear genome by reducing the amount of data used in the whole genome assembly that in some cases can reach $25 \%$ or more (e.g. chloroplast DNA in plants); 2) It can be used as a method to evaluate the relative quality of the PacBio sequencing data by assembling a small batch of reads.

\section{Conclusions}

Organelle_PBA is a program designed to assemble organelle genomes from PacBio whole genome sequencing data. Pre-selection of the organelle DNA sequencing reads using a mapping approach facilitates the organelle genome assembly and optimizes the computational requirements. It also removes the assembly redundancy caused by a circular assembly and resolves the chloroplast genome inverted repeats. Organelle_PBA performed 
successful assemblies of the mitochondrial and chloroplast genomes in model species such as $M$. musculus and $A$. thaliana respectively. The program was also used for the successful assembly of two novel chloroplast genomes from the species Picea glauca (a gymnosperm) and Sinningia speciosa (a eudicot angiosperm). The tool is freely available at https://github.com/aubombarely/Organelle_PBA.

\section{Availability and requirements}

- Project name: Organelle_PBA

- Project home page: https://github.com/aubombarely/ Organelle_PBA

- Archived version: Not applicable

- Operating system: Linux (tested on Ubuntu 14.04.1)

- Programming language: Perl

- Other requirements: Perl (5.18.2), BioPerl (1.6.924), Seqtk (1.0-r31), BlastN (2.3.0+), BlasR (1.3.1), Samtools (1.3-8-g03a6bc5), Bedtools (v2.17.0), Sprai (0.9.9.8), WGS-Assembler (8.3rc1), SSPACE-Long (v1-1).

- License: GNU General Public License

\section{Additional file}

Additional file 1: Figure S1. Chart flow for the Organelle_PBA software. Reads are mapped to an organelle reference using BlasR (1). The BlasR output is parsed and the sequence IDs are used to retrieve the reads from the input file (2). Organelle identified reads are assembled using Sprai (3). The program checks if the assembly is complete comparing its length with the reference (4). If it is not complete, it performs a scaffolding using SSPACE-Long and the whole PacBio dataset (5). It is complete it moves to the new checking point where it check for circularity (6). If it detects circularity by a self-BlastN, it trims the sequence corresponding to the circular overlap (6). Finally it check for the repeat assembly (7) and if it finds it, it breaks in four parts, identify the complete inverted repeat (IR), duplicate it (IRa and IRb) and re-assemble it will the long and short single copy (LSC, SSC) (9). (TIFF $5352 \mathrm{~kb}$ )

\section{Abbreviations}

DBG: De Bruijn Graph; InDel: Insertion-deletion; IR: Inverted repeat; LSC: Long single copy; OLC: Overlap-layout-consensus; SMRT: Single molecule real time; SNP: Single nucleotide polymorphism; SSC: Short single copy

\section{Acknowledgements}

This study was financially supported by the Plant and Microbial Genomic Cluster start up funds supplied by Virginia Tech.

\section{Funding}

The program and the experiments reported in this article were funded with start-up funds provided to Dr. Bombarely at Virginia Tech (Program: Plant and Genomic Cluster).

\section{Availability of data and materials}

The datasets supporting the conclusions in this article are available in the NCBI SRA repository:

\section{- For Arabidopsis thaliana:} http://www.ncbi.nlm.nih.gov/sra/?term=SRR1284093 http://www.ncbi.nlm.nih.gov/sra/?term=SRR1284094 http://www.ncbi.nlm.nih.gov/sra/?term=SRR1284095 http://www.ncbi.nlm.nih.gov/sra/?term=SRR1284703 http://www.ncbi.nlm.nih.gov/sra/?term=SRR1284704
- For Mus musculus: http://www.ncbi.n/m.nih.gov/sra/?term=ERR731675

- For Picea glauca: http://www.ncbi.nlm.nih.gov/sra/?term=SRR2148116

\section{Authors' contributions}

AS performed the experiments and reviewed the manuscript. DH and DZ reviewed the manuscript and contributed to the discussion of the experiments. $A B$ wrote the program, designed the experiments, wrote the manuscript, and discussed the design of the program and the results with the other contributors of the article. All authors read and approved the final manuscript.

\section{Competing interests}

The authors declare that they have no competing interests.

\section{Consent for publication}

Not applicable.

Ethics approval and consent to participate

Not applicable.

\section{Author details}

${ }^{1}$ Department of Horticulture, Virginia Polytechnic Institute and State University, Blacksburg, VA 24061, USA. ${ }^{2}$ Department of Horticulture, Faculty of Horticultural Sciences and Plant Protection, University of Tehran, Karaj 31587, Iran. ${ }^{3}$ Department of Plant Pathology, Physiology and Weed Science, Virginia Polytechnic Institute and State University, Blacksburg, VA 24061, USA. ${ }^{4}$ Kentucky Tobacco Research and Development Center (KTRDC), University of Kentucky, Lexington, KY 40546, USA.

Received: 22 April 2016 Accepted: 10 December 2016 Published online: 07 January 2017

\section{References}

1. Roberts RJ, Carneiro MO, Schatz MC. The advantages of SMRT sequencing Genome Biol. 2013;14:405.

2. Franzén O, Hu J, Bao X, Itzkowitz SH, Peter I, Bashir A. Improved OTUpicking using long-read $16 \mathrm{~S}$ rRNA gene amplicon sequencing and generic hierarchical clustering. Microbiome. 2015;3:43.

3. Sharon D, Tilgner $H$, Grubert F, Snyder M. A single-molecule long-read survey of the human transcriptome. Nat Biotech. 2013;31:1009-14.

4. Booher NJ, Carpenter SCD, Sebra RP, Wang L, Salzberg SL, Leach JE, Bogdanove AJ. Single molecule real-time sequencing of Xanthomonas oryzae genomes reveals a dynamic structure and complex TAL (transcription activator-like) effector gene relationships. Microb Genomics. 2015;1(4): doi:10.1099/mgen.0.000032.

5. Sakai H, Naito K, Ogiso-Tanaka E, Takahashi Y, Iseki K, Muto C, Satou K, Teruya K, Shiroma A, Shimoji M, Hirano T, Itoh T, Kaga A, Tomooka N. The power of single molecule real-time sequencing technology in the de novo assembly of a eukaryotic genome. Sci Rep. 2015;5:16780.

6. Chin C-S, Alexander DH, Marks P, Klammer AA, Drake J, Heiner C, Clum A, Copeland A, Huddleston J, Eichler EE, Turner SW, Korlach J. Nonhybrid, finished microbial genome assemblies from long-read SMRT sequencing data. Nat Meth. 2013;10:563-9.

7. Miller JR, Delcher AL, Koren S, Venter E, Walenz BP, Brownley A, Johnson J, Li K, Mobarry C, Sutton G. Aggressive assembly of pyrosequencing reads with mates. Bioinformatics. 2008;24:2818-24

8. Berlin K, Koren S, Chin C-S, Drake JP, Landolin JM, Phillippy AM. Assembling large genomes with single-molecule sequencing and locality-sensitive hashing. Nat Biotechnol. 2015;33:623-30.

9. Miyamoto M, Motooka D, Gotoh K, Imai T, Yoshitake K, Goto N, lida T, Yasunaga T, Horii T, Arakawa K, Kasahara M, Nakamura S. Performance comparison of second- and third-generation sequencers using a bacterial genome with two chromosomes. BMC Genomics. 2014;15:699.

10. McGinnis S, Madden TL. BLAST: at the core of a powerful and diverse set of sequence analysis tools. Nucleic Acids Res. 2004

11. Wolters JF, Chiu K, Fiumera HL. Population structure of mitochondrial genomes in Saccharomyces cerevisiae. BMC Genomics. 2015;16:451.

12. Ferrarini M, Moretto M, Ward JA, Šurbanovski N, Stevanović V, Giongo L, Viola R, Cavalieri D, Velasco R, Cestaro A, Sargent DJ. An evaluation of the 
PacBio RS platform for sequencing and de novo assembly of a chloroplast genome. BMC Genomics. 2013;14:670.

13. Wu Z, Gui S, Quan Z, Pan L, Wang S, Ke W, Liang D, Ding Y. A precise chloroplast genome of Nelumbo nucifera (Nelumbonaceae) evaluated with Sanger, Illumina MiSeq, and PacBio RS II sequencing platforms: insight into the plastid evolution of basal eudicots. BMC Plant Biol. 2014;14:289.

14. Stadermann KB, Weisshaar B, Holtgräwe D. SMRT sequencing only de novo assembly of the sugar beet (Beta vulgaris) chloroplast genome. BMC Bioinformatics. 2015;16:295.

15. Avise JC, Arnold J, Martin Ball R, Bermingham E, Lamb T, Neigel JE, Reeb CA, Saunders NC. Intraspecific phylogeography: the mitochodrial DNA bridge between population genetics and systematics. Annu Rev Ecol Syst. 1987;18: 489-522.

16. Kurabayashi A, Sumida M. Afrobatrachian mitochondrial genomes: genome reorganization, gene rearrangement mechanisms, and evolutionary trends of duplicated and rearranged genes. BMC Genomics. 2013;14:633.

17. Smith KF, Abbott CL, Saito Y, Fidler AE. Comparison of whole mitochondrial genome sequences from two clades of the invasive ascidian, Didemnum vexillum. Mar Genomics. 2015;19:75-83.

18. Vahrenholz C, Riemen G, Pratie E, Dujon B, Michaelis G. Mitochondrial DNA of Chlamydomonas reinhardtii: the structure of the ends of the linear 15.8-kb genome suggests mechanisms for DNA replication. Curr Genet. 1993;24:241-7.

19. Sloan DB, Alverson AJ, Chuckalovcak JP, Wu M, McCauley DE, Palmer JD, Taylor DR. Rapid evolution of enormous, multichromosomal genomes in flowering plant mitochondria with exceptionally high mutation rates. PLoS Biol. 2012:10:e1001241.

20. Bellot S, Renner SS. The plastomes of two species in the Endoparasite Genus Pilostyles (Apodanthaceae) each retain just five or six possibly functional genes. Genome Biol Evol. 2016;8:189-201.

21. Brouard J-S, Otis C, Lemieux C, Turmel M. The exceptionally large chloroplast genome of the green alga Floydiella terrestris illuminates the evolutionary history of the Chlorophyceae. Genome Biol Evol. 2010;2:240-56.

22. Zaitlin D. Intraspecific diversity in Sinningia speciosa (Gesneriaceae: Sinningieae), and possible origins of the cultivated florist's gloxinia. AoB Plants. 2012;2012:pls039. doi:10.1093/aobpla/pls039.

23. Chaisson MJ, Tesler G. Mapping single molecule sequencing reads using basic local alignment with successive refinement (BLASR): application and theory. BMC Bioinformatics. 2012;13:238.

24. Li H, Handsaker B, Wysoker A, Fennell T, Ruan J, Homer N, Marth G, Abecasis G, Durbin R, 1000 Genome Project Data Processing Subgroup. The sequence alignment/map format and SAMtools. Bioinformatics. 2009;25:2078-9.

25. Quinlan AR, Hall IM. BEDTools: a flexible suite of utilities for comparing genomic features. Bioinformatics. 2010;26:841-2.

26. Garrison E, Marth G. Haplotype-based variant detection from short-read sequencing. arXiv. 2012. arXiv:1207.3907.

27. Boetzer M, Pirovano W. SSPACE-LongRead: scaffolding bacterial draft genomes using long read sequence information. BMC Bioinformatics. 2014;15:1.

\section{Submit your next manuscript to BioMed Central and we will help you at every step:}

- We accept pre-submission inquiries

- Our selector tool helps you to find the most relevant journal

- We provide round the clock customer support

- Convenient online submission

- Thorough peer review

- Inclusion in PubMed and all major indexing services

- Maximum visibility for your research

Submit your manuscript at www.biomedcentral.com/submit

) Biomed Central 